\title{
Primary reference in terms of air kerma for ionizing radiation fields produced by an electrostatic electron accelerator
}

\author{
Jean-Marc Bordy ${ }^{*}$, Valentin Blideanu ${ }^{1}$, Arnaud Chapon ${ }^{2}$, Gabriel Dupont ${ }^{2}$, Dorin \\ Dusciac $^{1}$, Jean Gouriou ${ }^{1}$, Frederic Laine ${ }^{3}$, and Maiwenn Le Roy ${ }^{1}$ \\ ${ }^{1}$ CEA, LIST, Laboratoire National Henri Becquerel (LNE-LNHB), CEA-Saclay, 91191 Gif sur \\ Yvette Cedex, France \\ ${ }^{2}$ ATRON METROLOGY, 14 allée des vindits, parc d'activité des fourches, 50130 Cherbourg en \\ Cotentin, France \\ ${ }^{3}$ CEA, LIST, DM2I SCI LCAE, CEA-Saclay, 91191 Gif sur Yvette Cedex, France
}

\begin{abstract}
Based on a radiation field produced by an electrostatic accelerator, a radiation survey meter test and calibration facility has been designed and characterized in terms of air kerma and ambient dose equivalent. The electron beam impinges a tantalum target to produce $\mathrm{X}$-rays. The spectrum has been measured and calculated. Traceability to the International System of units is achieved by means of a calibration with a primary dosimeter for air kerma.
\end{abstract}

\section{INTRODUCTION}

The French legislation has introduced a maximum period of use for ionization radiation isotopic sources; beyond 10 years these must be changed or can be requalified for a limited period. To reduce the operating costs of reference and calibration facilities and to facilitate calibration procedures, the idea came to produce bremsstrahlung photon radiation fields from linear electron accelerators. A first realization was made at CEA/LNE-LNHB, based on a medical LINAC Saturne 43, leading to the characterization in terms of air kerma and dose equivalent of a radiation field with an average X-ray energy of about $7 \mathrm{MeV}$ for an initial electron beam of $18 \mathrm{MeV}$ [1].

CEA/LNE-LNHB characterized a facility designed by the private company ATRON METROLOGY for the test and calibration of radiation survey meters in terms of air kerma and ambient dose equivalent. This facility, based on the same principle, targets an energy domain between ${ }^{137} \mathrm{Cs}$ and ${ }^{60} \mathrm{Co}$. This publication describes the technological choices of ATRON METROLOGY and the entire process of (i) the primary characterization of ATRON METROLOGY beams in terms of air kerma and (ii) the establishment of conversion coefficients from this quantity to the ambient dose equivalent by CEA/LNE-LNHB.

\section{ATRON METROLOGY FACILITY}

ATRON METROLOGY chose an electrostatic Singletron accelerator from HVE (High Voltage Engineering Europa B.V., Amersfoort, Netherlands). It delivers a continous beam of electrons, the maximum high voltage is $3.5 \mathrm{MV}$. The $22 \times 4 \mathrm{~cm}^{2}$ target is made of a tantalum plate $\left({ }^{73} \mathrm{Ta}\right), 1.5 \mathrm{~mm}$ thick. The electron beam scans this plate. The current range lie from a 
few $0.1 \mathrm{pA}$ to $1 \mathrm{~mA}$ [2]. Within a range between $200 \mathrm{kV}$ and $3.5 \mathrm{MV}$, the accelerator has three pre-settings in energy $1.25 \mathrm{MeV}, 2 \mathrm{MeV}$ or $3 \mathrm{MeV}$.

ATRON METROLOGY developed, in collaboration with the Laboratoire de Physique Corpusculaire de Caen (LPC Caen - ENSICAEN/UNICAEN/CNRS): (i) a transmission ionization chamber to regulate the electron beam at low dose rates, (ii) a sample conveyor positioned at a distance of 1 or 3 meters from the target. Two cavity chambers PTW TM32002 (1 L) associated with PTW UNIDOS Webline T10021 electrometers are fixed on the latter in the photon beam for monitoring (Figure 1).

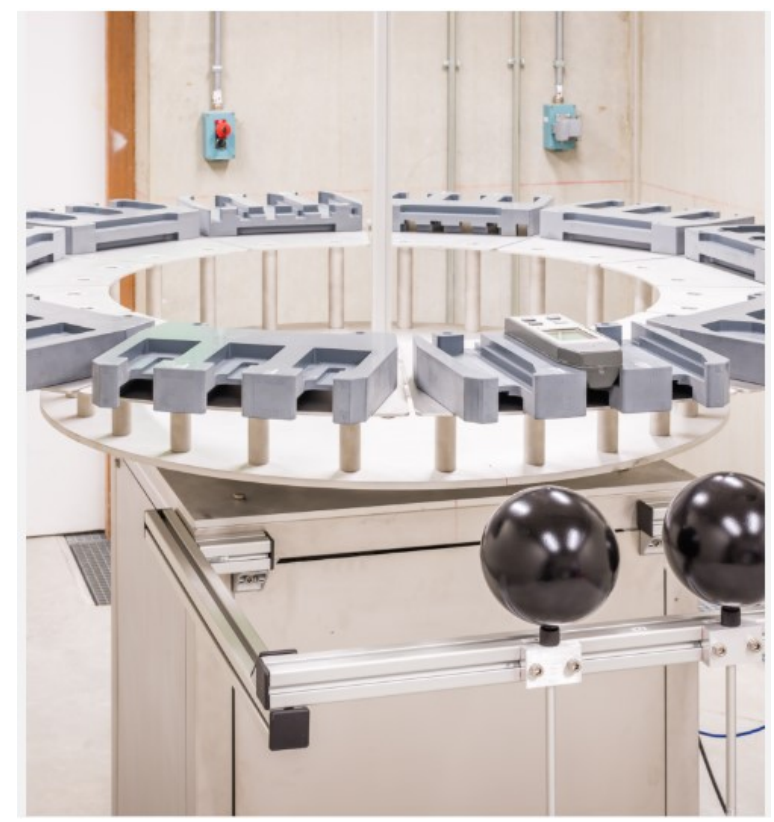

Fig 1. Picture of the sample conveyor with the two monitoring chambers at the front

\section{SPECTROMETRY}

To establish a reference in terms of air kerma and dose equivalent, it is essential to know the spectrum of photons at the measurement point in order to calculate: (i) the correction factors to be applied to the ionization chamber primary measurement of air kerma, (ii) the conversion coefficient from air kerma into ambient dose equivalent. The following method is used to determine the spectrum at the measurement point:

a) Calculate the "spectrum" at the measurement point in air (MCNPX) [3],

b) Measure the spectrum at the measurement point with a BGO 3" scintillator,

c) Calculate the pulse distribution of the spectrometer (MCNPX),

d) Compare of the calculated pulse distribution versus the measured one.

The comparison of the pulse distributions ( $\mathrm{b} \& \mathrm{c}$ ) validates the use of the code therefore the calculation of the spectra (a). Figure 2 shows the spectrum calculated at $1 \mathrm{~m}$ from the target for a high voltage of $3 \mathrm{MV}$. The average energy of the spectrum is $0.64 \mathrm{MeV}$.

Figure 3 shows the comparison between the spectra measured and calculated for the configuration "1 m, $2 \mathrm{MeV}$ ". An energy threshold is applied to limit the influence of dead time on the measurement. The surface of the spectra is normalized to the unity. In order to facilitate the graphical comparison of spectrum shapes between experimental and computational results, scaling factors were applied to one of the spectra. 


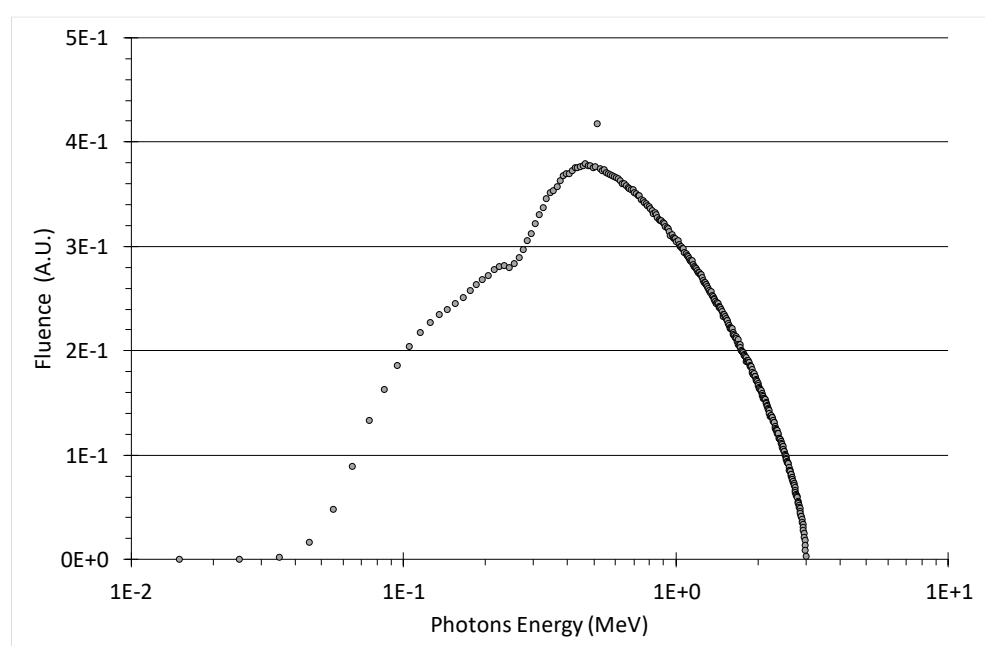

Fig 2. Fluence energy distribution for the configuration 1m, 3 MV calculated with MCNP "tally 5".

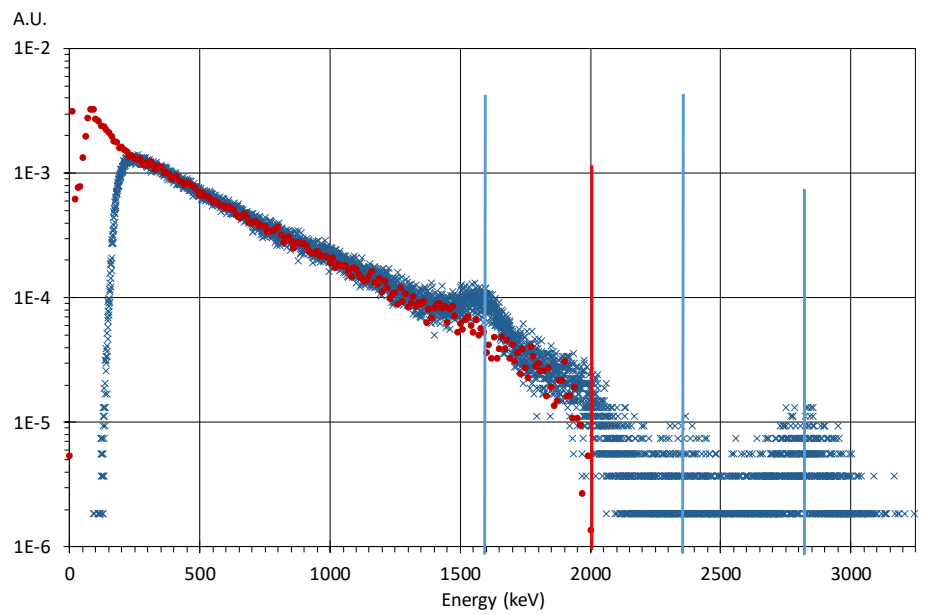

Fig 3. Comparison of the calculated (red) and measured (blue) spectra, configuration 1m, $2 \mathrm{MeV}$.

There are three peaks around $1600 \mathrm{keV}, 2350 \mathrm{keV}$ and $2800 \mathrm{keV}$ on the measured spectrum. The origin of these peaks was searched using the radionuclides library of photon emissions published by CEA/LNE-LNHB (www.Nucleide.org/LARAWEB). Considering the energy resolution of the spectrometer (about $16 \%$ for ${ }^{60} \mathrm{Co}$ ) and the radioactive decays of the nuclides emitting in these energy domains, ${ }^{226} \mathrm{Ra}$ appears to be the best candidate but the measurement environment does not allow assigning these peaks to one nuclide, nevertheless potassium 40 ( $1460.8 \mathrm{keV}, 10.6 \%$ intensity) may also contribute to the $1600 \mathrm{keV}$ peak.

The pulse distribution calculation was performed using a tally type F8 (energy deposit in the volume of the BGO scintillator). Outside the "threshold" zone, the adequacy between the shape of the calculated and measured pulse distribution is good. Therefore, it can be concluded that the code and the implemented geometry are validated for the ATRON METROLOGY configurations and so the spectrum of the figure 2 can be used to calculate the correction factors and conversion coefficients. These three peaks found on the measured spectrum are at least two decades lower than the main component of the spectrum so with no significant weight on the further conversion coefficient and the correction factors calculations. 


\section{TRACEABILITY TO THE INTERNATIONAL SYSTEM OF UNITS}

In this section, « $\mathrm{AM}$ » stands for "ATRON METROLOGY radiation field" and «LNHB » for "CEA/LNE LNHB Co/Cs beams". When a device is verified or calibrated in the AM radiation field, the value of the dose equivalent at the measurement point is calculated as follows:

$$
H^{*}(10)=K_{a, A M} \cdot \overline{h_{k, A M}^{*}}
$$

with $\overline{h_{k, A M}^{*}}$, the average conversion coefficient between air kerma and ambient dose equivalent for the AM spectrum. It is calculated using the conversion coefficients, $h_{k}^{*}$, published in the ICRU57 report for discrete energies, weighted by the energy distribution of the photon fluence. $\Phi_{A M}$. The untabulated values of these conversion coefficients are calculated using a 4-point Lagrangian log-log interpolation.

$$
\overline{h_{k, A M}^{*}}=\frac{\int h_{k}^{*}(E) E \Phi_{A M}(E) d E}{\int E \Phi_{A M}(E) d E}=1.20 S v / G y
$$

This value, calculated for the " $1 \mathrm{~m}, 3 \mathrm{MV}$ " configuration, is close to the ${ }^{137} \mathrm{Cs}$ one published in ISO 4037, 1.21 Sv/Gy. The air kerma in the AM fields is:

$$
K_{a, A M}=I_{\text {Monitor }} . N_{K_{a}, \text { Monitor }, A M}
$$

$\mathrm{I}_{\text {Monitor }}$ is the monitor reading corrected for ambient conditions,

$\mathrm{N}_{\mathrm{Ka} \text {,Monitor,AM }}$ is the calibration coefficient of the monitor in terms of air kerma in the AM radiation field. It can be determined directly through a primary measurement of air kerma in the AM radiation field or indirectly through the calibration of the monitor in terms of air kerma in the LNHB calibration beams $\left({ }^{60} \mathrm{Co}\right.$ or $\left.{ }^{137} \mathrm{Cs}\right)$ multiplied by a radiation quality conversion factor, $k_{L N H B, A M}$, in terms of air kerma, from the CEA/LNE LNHB beams to the $\mathrm{AM}$ radiation field. This last conversion factor is derived from the ratio of the calibration factors of the monitor in AM and LNHB radiation fields.

$$
N_{K_{a}, \text { Monitor }, A M}=N_{K_{a}, \text { Monitor }, L N H B} \cdot k_{L N H B, A M}
$$

The traceability of the AM dose equivalent to the SI is therefore achieved through a CEA/LNE-LNHB primary measurement on site or through the CEA/LNE-LNHB national references.

\section{PRIMARY STANDARD}

An ionization chamber was designed and manufactured by CEA/LNE-LNHB. Hereafter one described shortly (i) the design of this chamber, (ii) the calculation of the collection volume and (iii) the electrical tests. The design of the chamber is identical to that of the chambers developed by the CEA/LNE-LNHB for its own needs (Figure 4). A wall thickness of $3 \mathrm{~mm}$ allows reaching electronic equilibrium - this point was confirmed during on-site measurements by adding PMMA filters of variable thickness. 


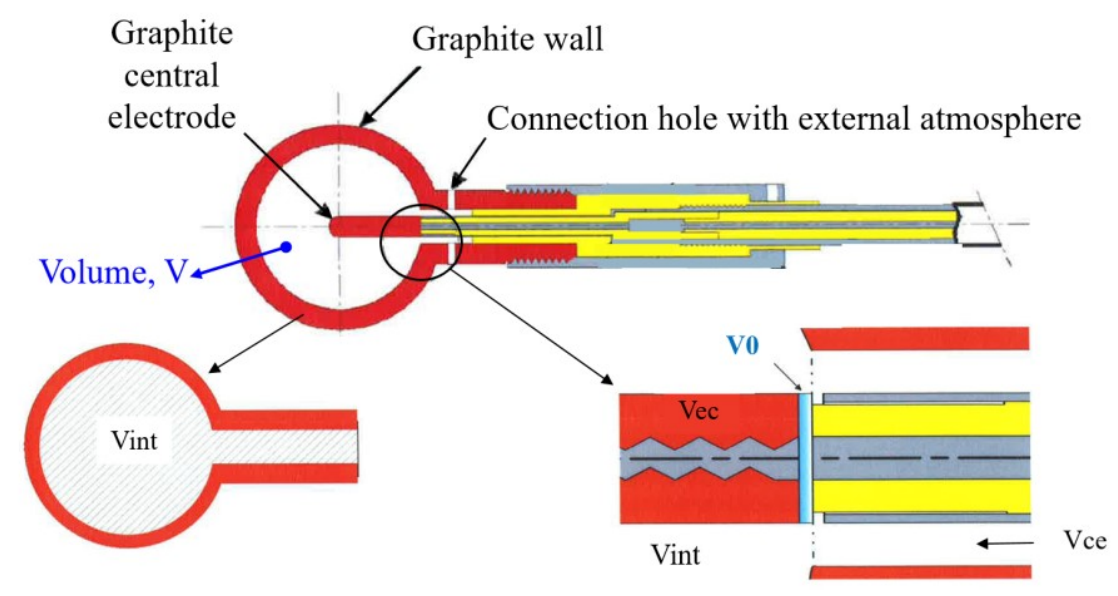

Fig 4. Cut view of the CEA/LNE-LNHB ionisation chamber, $\mathrm{red} /$ graphite, yellow/insulator and grey/metal.

The effective charge collection volume, $\mathrm{V}$, is smaller than the measured cavity volume Vint (as described in [5]). One has to subtract, (i) Vec, the inner electrode volume, (ii) Vce, the volume part of the stick, and (iii) V0, the volume just below the inner electrode and out of the stick. The effective collection volume is $7.1735(0.0042) \mathrm{cm}^{3}$. All the volumes are calculated from geometric measurements made at the LNE. Once cleaned, the parts are assembled and the electrical test is carried out. Its aim is to define the operating point in ionization chamber mode and to verify that the correction factors for saturation $\mathrm{k}_{\mathrm{s}}$ and polarity $\mathrm{k}_{\mathrm{pol}}$ are compatible with a metrological use.

The stability of the current measured under irradiation over a period of 7 hours shows a very good stability with a maximum variation of about $0.02 \%$. The measured leakage current is in the range of $10^{-16}-10^{-15} \mathrm{~A}$. The variation of the current as a function of the polarization voltage of the chamber is shown in Figure 5. The operating point, $+850 \mathrm{~V}$, allows an optimal collection without approaching the multiplication zone.

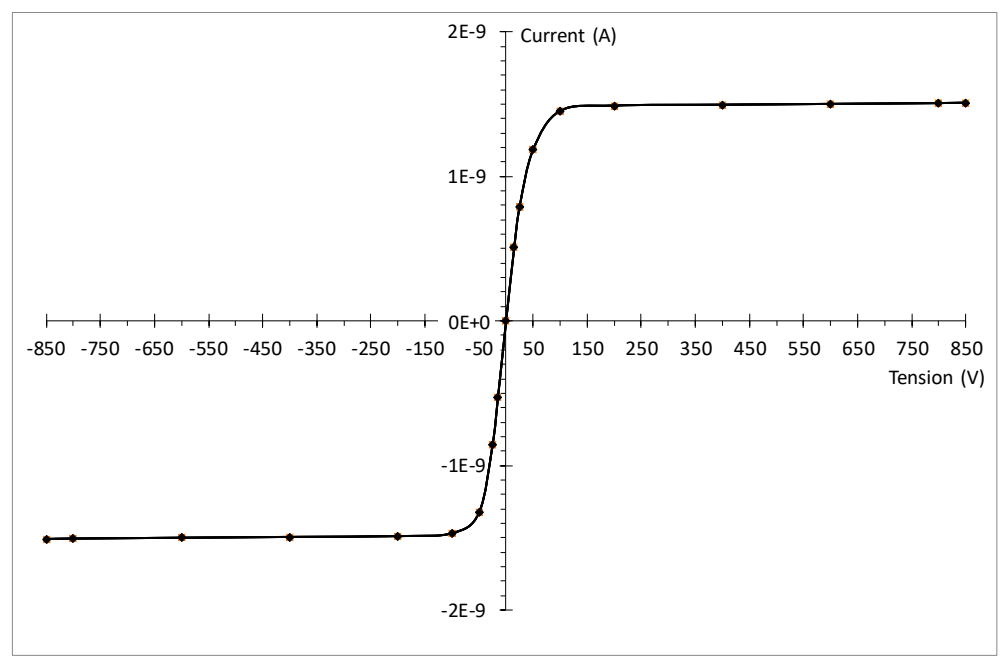

Fig 5. Variation of the current as a function of the high voltage applied to the chamber. 
The correction factor for polarity is:

$$
k_{p o l}=\frac{I_{c o r,+850 V}-I_{c o r,-850 V}}{2 I_{c o r}+850 \mathrm{~V}}
$$

The correction factor for the saturation (initial in our case) is measured using the two voltages method with $\mathrm{V}_{1}=\mathrm{V}_{0} / 2=425 \mathrm{~V}, \mathrm{I}_{1}$ and $\mathrm{I}_{0}$ are the corrected currents measured with the two voltages respectively and corrected for polarity at their respective voltages.

$$
k_{S}\left(V_{0}\right)=\frac{I_{\infty}}{I_{0}}=1+\frac{\frac{I_{0}}{I_{1}}-1}{\frac{V_{0}}{V_{1}}-\frac{I_{0}}{I_{1}}}
$$

The associated uncertainties are:

$$
\frac{u\left(k_{\text {pol }}\right)}{k_{\text {pol }}}=\left(\frac{\sqrt{2}}{2} \frac{u\left(I_{\text {cor }}\right)}{I_{\text {cor }}}\right) / k_{\text {pol }} \quad \frac{u\left(k_{s}\right)}{k_{s}}=\left(\sqrt{2} \frac{u\left(I_{\text {cor }}\right)}{I_{\text {cor }}}\right) / k_{S}
$$

The values of $\mathrm{k}_{\mathrm{pol}}$ and $\mathrm{k}_{\mathrm{s}}$ are given in Table 1 with their standard uncertainties in brackets. In all cases encountered, the signal-to-leakage current ratio is always at least in the order of 10000 , so the influence of uncertainties associated with measurements of the leakage current on the final result is negligible.

Table $1 . \mathrm{ks}_{\mathrm{s}}$ and $\mathrm{kpol}_{\mathrm{v}}$ values.

\begin{tabular}{|c|c|c|}
\hline $\mathrm{k}_{\mathrm{pol}}+850 \mathrm{~V}$ & $\mathrm{k}_{\mathrm{pol}}+425 \mathrm{~V}$ & $\mathrm{k}_{\mathrm{s}}$ initial \\
\hline $1.000235(12)$ & $0.999955(13)$ & $1.001740(14)$ \\
\hline
\end{tabular}

The air kerma measurements were carried out at the premises of ATRON METROLOGY. The formula for calculating the air kerma from the ionization chamber indication is as follows:

$$
K_{\text {alr }}^{\cdot}=\frac{I_{\text {cor }}}{V \rho_{\text {air }}} \frac{1}{A_{\text {wall }}}(W / e)_{\text {air }}\left(\frac{\bar{S}}{\rho}\right)_{\text {graph,air }}\left(\frac{\overline{\mu_{e n}}}{\rho}\right)_{\text {air,graph }} \frac{1}{1-\overline{g_{\text {alr }}}}
$$

$\boldsymbol{K}_{\boldsymbol{a}}^{\cdot}$ is the air kerma rate normalized to monitor,

$\mathrm{I}_{\mathrm{cor}}$ is the current corrected for ambient condition and background normalized to monitor, $\rho_{\text {air }}$ is the dry air density at $293.15 \mathrm{~K}$ and $1013.25 \mathrm{hPa}$,

$\mathrm{V}$ is the effective collection volume,

$(\mathrm{W} / \mathrm{e})_{\text {air }}$ is the average energy to produce an ion-electron pair divided by the electron charge, $(\overline{\boldsymbol{S} / \boldsymbol{\rho}})_{\text {graph,air }}$ is the ratio of the fluence weighted average values of the mass stopping power between graphite and air,

$\left(\overline{\mu_{e n} / \rho}\right)_{\text {air,graph }}$ is the ratio of the energy fluence weighted average values of the mass energy absorption coefficient between air and graphite. The $\mu_{\mathrm{en}} / \rho$ values are taken from NIST web site (WWW.nist.gov),

$\overline{\boldsymbol{g}_{\boldsymbol{a}} \boldsymbol{r}}$ is the average fraction of the initial electron energy lost with radiative processes in air, $\mathrm{A}_{\text {wall }}$ is the correction for the attenuation and diffusion in the chamber wall. 
The corrected current is:

$$
I_{c o r}=\frac{\left(I_{\text {raw }, I C}-I_{B G, I C}\right) k_{H} k_{r e c} k_{\text {pol }} k_{\text {stick }} k_{\text {capa }}}{\left(I_{\text {raw }, \text { mon }}-I_{B G, \text { mon }}\right)}
$$

$\mathrm{I}_{\mathrm{raw}, \mathrm{XX}}$ is the average value of the current at the measurement point, $\mathrm{I}_{\mathrm{BG}, \mathrm{XX}}$ is the average value of the leakage current when the beam is off,

The subscript "IC" stands for "primary ionization chamber" whereas the subscript "mon" stands for "monitor chamber", $\mathrm{k}_{\mathrm{H}}$ is the correction factor for humidity, $\mathrm{k}_{\text {stick }}$ is the correction factor the radiation scattered by the chamber stick, $\mathrm{k}_{\text {capa }}$ is the correction factor to account for the capacitor sensitivity to the temperature.

The combined relative uncertainty on the air kerma rate normalized to the monitor reading is equal to the square root of the quadratic sum of the relative uncertainties of the correction factors and physical parameters following the Guide to the expression of Uncertainty [4]. The standard uncertainties associated with the parameters $\left(\overline{\mu_{e n} / \rho}\right)_{\text {air,graph }},(\overline{S / \rho})_{\text {graph,air }}$ and $\overline{1-g_{\text {arr }}}$ are envelope values estimated by taking into account the uncertainty on the spectrum and the ones associated with the discrete values of the interaction coefficients. The table 2 presents the values of the physical parameters used to determine the air kerma rate according to formula ( 8 and 9 ) and their associated uncertainties.

Table 2. Air kerma and ambient dose equivalent values with associated uncertainty.

\begin{tabular}{|c|c|c|}
\hline & Value & Standard uncertainty \\
\hline $\mathrm{I}_{\text {cor }} /$ Monitor & $-1.047510^{-4}$ & $0.405 \%$ \\
\hline Volume $\left(\mathrm{cm}^{3}\right)$ & 7.1735 & $0.0042 \%$ \\
\hline Masse volumique de l'air $\left(\mathrm{g} / \mathrm{cm}^{3}\right)$ & $1.204710^{-03}$ & $0.001 \%$ \\
\hline $\mathrm{k}_{\mathrm{pol}}$ & 1.0017 & $0.286 \%$ \\
\hline $\mathrm{k}_{\mathrm{s}}$ & 0.9994 & $0.573 \%$ \\
\hline $\mathrm{k}_{\text {stick }}$ & 0.9983 & $0.257 \%$ \\
\hline A wall $_{1}$ & 0.9798 & $0.200 \%$ \\
\hline$\left(\overline{\mu_{e n} / \rho}\right)_{a i r, g r a p h}$ & 0.9975 & $1.000 \%$ \\
\hline$(\overline{S / \rho})_{\text {graph }, a i r}$ & 0.9979 & $1.000 \%$ \\
\hline$(W / e)_{\text {air }}(\mathrm{J} / \mathrm{C})$ & 33.97 & $0.350 \%$ \\
\hline$\overline{1-g_{\text {air }}}$ & 0.9968 & $1.400 \%$ \\
\hline$K_{\text {alr }}^{\cdot} /$ Monitor (Gy/M.U.) & $2.51610^{4}$ & $2.18 \%$ \\
\hline$H^{*}(10) / K_{\text {alr }}^{*}(\mathrm{~Sv} / \mathrm{Gy})$ & 1.20 & $0.3 \%$ \\
\hline$H^{*}(10) /$ Monitor (Sv/ M.U.) & $3.0210^{4}$ & $2.2 \%$ \\
\hline
\end{tabular}

M.U. monitor unit 


\section{CONCLUSIONS}

An innovative way to meet the needs of nuclear industry in terms of radiation survey meter verification and calibration services was developed through an efficient collaboration between the French national metrology laboratory (CEA/LNE-LNHB) and industry (ATRON METROLOGY). The facility allows changing the dose equivalent rate without moving the dosimeters just by tuning the electron beam intensity over 8 decades and can automatically calibrate up to 32 devices carried by a coveyor. The traceability to the international system of units is achieved through a primary measurement of the air kerma onsite and a regular calibration/verification in front of the French national references of CEA/LNE-LNHB.

\section{References}

[1] D. Dusciac, J.-M. Bordy, J. Daures and V. Blideanu, EPJ Web of Conferences, DOI: http://dx.doi.org/10.1051/epjconf/201612400004, 124 (2016),

[2] A. Chapon - G. Dupont - J.M. Bordy, Revue Générale Nucléaire, 6, 48-52 (2016).

[3] G. Pelowitz, MCNPX user's manual, version 2.5.0 (2005)

[4] ISO 13005, Guide pour l'expression des incertitudes de mesures, (1999).

[5] F Delaunay, M Donois, J Gouriou, E Leroy and A Ostrowsky, New LNHB primary standard for 60Co air kerma Metrologia 47 (2010) 652, https://doi.org/10.1088/0026$1394 / 47 / 6 / 004$ 